\title{
Integrability and Hopf Solitons in Models with Explicitly Broken $O(3)$ Symmetry
}

\author{
A. Wereszczyński ${ }^{a)}$ * \\ a) Institute of Physics, Jagiellonian University, \\ Reymonta 4, Kraków, Poland
}

December 25, 2018

\begin{abstract}
A wide class of models, built of the three component unit vector field living in the $(3+1)$ Minkowski space-time, which break explicitly global $O(3)$ symmetry are discussed. The symmetry breaking occurs due to the so-called dielectric function multiplying a standard symmetric term. Integrability conditions are found. Moreover, for some particular forms of the Lagrangian exact toroidal solutions with any Hopf index are obtained. It is proved that such symmetry breaking influences the shape of the solitons whereas the energy as well as the Hopf index remain unchanged.
\end{abstract}

*wereszczynski@th.if.uj.edu.pl 


\section{Introduction}

Since Faddeev, Niemi [1], 2] and Cho [3] have proposed their famous action, which is considered as a good candidate for an effective model describing the non-perturbative regime of the gluodynamics, toroidal topological field configurations i.e. toroidal solitons have been widely investigated [4, [5], 6], [7, [8. They give a very attractive framework to understand the physics of the glueballs - particles maid only of the gauge field. In this picture a particular glueball can be described by a toroidal soliton with the pertinent topological number, so-called Hopf index [9], [10]. It is in the agreement with the standard picture of the mesons, where quarks are connected by a very thin tube of the gauge field. Now, because of the fact that glueballs do not consist of quarks, such a flux-tube cannot end on the sources. In order to form a stable object the ends must be joined giving loop-like configurations. However, in spite of the fact that Faddeev-Niemi model gives us the chance for the very elegant way to describe physics of the glueballs, it has also its own problems. One of the most important problems is the existence of massless excitations, that is Goldstone bosons which appear as the effect of the spontaneous symmetry breaking. In fact, the Lagrangian has global $O(3)$ symmetry whereas the vacuum state is only $O(2)$ invariant. Thus, two generators are broken and two bosons emerge. This feature of Faddeev-Niemi model has been recently discussed and some modifications have been proposed [11, [12], 13], 14]. In general, they mean necessity of the adding new terms into the Lagrangian, which assure the explicit $O(3)$ symmetry breaking.

The main aim of the present work is to analyze toroidal solitons in models with explicitly broken $O(3)$ symmetry. Such problems as existence of knotted solitons in these models, influence of symmetry breaking term on energy and shape of knots have been not analyzed yet. In this paper it will be shown that unbroken $O(3)$ global symmetry is not necessary condition for existence of knots. Of course, there are infinitely many way in which the symmetry can be broken at the Lagrangian level. Here, the simplest case is chosen - a symmetric part of the model is multiplied by some function, usually called dielectric function, which explicitly breaks the symmetry. However, such simple pattern of the symmetry breaking is strongly motivated by the latest investigation of the effective action for the low energy QCD. Namely, using the non-Abelian color dielectric model one can derive, in the limit when the color dielectric field condenses, a modified version of the Faddeev-Niemi action [14], 15]. The modification is given by two functions which multiply the standard Faddeev-Skyrme and kinetic terms. These functions depend only on the unit vector field and break explicitly $O(3)$ symmetry. One can 
immediately ask about the fate of the toroidal solutions in the theories with explicitly broken $O(3)$ symmetry. Unfortunately, obtained model is even more complicated than the standard Faddeev-Niemi model and none analytical solutions are known. In spite of that, this problem can be investigated and solved in case of the toy model which is defined below.

To conclude, the question how the explicit breaking of the global $O(3)$ symmetry modifies the topological toroidal solitons deserves answer. It is clear that results obtained here do not have to concern the modified versions of the Faddeev-Niemi model. Nonetheless, some effects seem to be quite general and might appear in some modified models [14 as well. Thus, one can treat this paper as the first step to understand what happen with hopfions in the modified Faddeev-Niemi theories.

\section{The Model}

Let us start with the Aratyn-Ferreira-Zimerman model [16], 17]

$$
\mathcal{L}=\left[\left[\vec{n} \cdot\left(\partial_{\mu} \vec{n} \times \partial_{\nu} \vec{n}\right)\right]^{2}\right]^{\frac{3}{4}},
$$

where $\vec{n}$ is a three component unit vector leaving in the Minkowski spacetime.

In our work we generalize it to the following Lagrangian density

$$
\mathcal{L}=\sigma(\vec{n})\left[\left[\vec{n} \cdot\left(\partial_{\mu} \vec{n} \times \partial_{\nu} \vec{n}\right)\right]^{2}\right]^{\frac{3}{4}}
$$

The symmetry breaking occurs due to the so-called dielectric function $\sigma$, which depends on the unit field only. On the other hand, the symmetric part can be written as a function of antisymmetric field tensor $H_{\mu \nu}=\vec{n} \cdot\left(\partial_{\mu} \vec{n} \times\right.$ $\left.\partial_{\nu} \vec{n}\right)$. The unit field can be, in the standard way, expressed by a complex field $u, u^{*}$ :

$$
\vec{n}=\frac{1}{1+|u|^{2}}\left(u+u^{*},-i\left(u-u^{*}\right),|u|^{2}-1\right) .
$$

Then we obtain

$$
\mathcal{L}=\sigma\left(u, u^{*}\right) \frac{8^{3 / 4}}{\left(1+|u|^{2}\right)^{3}}\left(K_{\mu} \partial^{\mu} u^{*}\right)^{\frac{3}{4}}
$$

where following [16] we define $K_{\mu}=\left(\partial_{\nu} u^{*} \partial^{\nu} u\right) \partial_{\mu} u-\left(\partial_{\nu} u \partial^{\nu} u\right) \partial_{\mu} u^{*}$. This object has two important properties:

$$
K_{\mu} \partial^{\mu} u=0 \quad \text { and } \quad \operatorname{Im}\left(K_{\mu} \partial^{\mu} u^{*}\right)=0
$$


The pertinent equations of motion read

$$
\partial_{\mu} \frac{3}{2}\left[\frac{\sigma}{\left(1+|u|^{2}\right)^{3}}\left(K_{\mu} \partial^{\mu} u^{*}\right)^{-\frac{1}{4}} K^{\mu}\right]-\left(K_{\mu} \partial^{\mu} u^{*}\right)^{\frac{3}{4}}\left[\frac{\sigma}{\left(1+|u|^{2}\right)^{3}}\right]_{u^{*}}^{\prime}=0
$$

and its complex conjugation. One can rewrite it into the more compact form

$$
\partial_{\mu}\left[\frac{\sigma^{1 / 3}}{\left(1+|u|^{2}\right)}\left(K_{\mu} \partial^{\mu} u^{*}\right)^{-\frac{1}{4}} K^{\mu}\right]=0
$$

or

$$
\partial_{\mu} \mathcal{K}^{\mu}=0
$$

where

$$
\mathcal{K}_{\mu}=\frac{\sigma^{1 / 3}}{\left(1+|u|^{2}\right)}\left(K_{\mu} \partial^{\mu} u^{*}\right)^{-\frac{1}{4}} K^{\mu}
$$

One can check that $\mathcal{K}$ fulfills the properties (15) only if the dielectric function is a real function

$$
\sigma=\sigma^{*}
$$

After that we are able to define an infinite family of the conserved currents using the procedure proposed in [16]. Namely,

$$
J_{\mu} \equiv \mathcal{K}_{\mu} \frac{\partial G}{\partial u}-\mathcal{K}_{\mu}^{*} \frac{\partial G}{\partial u^{*}}
$$

where $G=G\left(u, u^{*}\right)$. Thus, the analyzed model is integrable. Integrability is understood as existence of the infinite number of the conserved currents [18, [19. It is straightforward to check that the integrability is observed for all models where the symmetric part is any function of the field tensor $H_{\mu \nu}^{2}$. The case considered here has been chosen to omit the Derick theorem for nonexistence of stable solitons. Thus, we see that integrability property is not connected with the global $O(3)$ symmetry. It is rather unexpected result. Usually, integrability (and solitons) are observed in maximally symmetric situation.

Now, we show that, as in the standard theories with solitons, integrability leads to appearance of soliton solutions.

Because of the fact that our aim is to find topological toroidal solitons with the non-trivial Hopf number we introduce the toroidal coordinates

$$
\begin{aligned}
& x=\frac{a}{q} \sinh \eta \cos \phi, \\
& y=\frac{a}{q} \sinh \eta \sin \phi,
\end{aligned}
$$




$$
z=\frac{a}{q} \sin \xi
$$

where $q=\cosh \eta-\cos \xi$ and $a>0$ is a constant of dimension of length fixing the scale in the coordinates. Moreover, we assume the following Ansatz for the field $u$ [16]

$$
u(\eta, \xi, \phi) \equiv f(\eta) e^{i(m \xi+n \phi)},
$$

where $m, n$ are integers.

Then, the static equation of motion takes the form

$$
\partial_{\eta} \ln \frac{\sigma^{2 / 3} f f^{\prime}}{\left(1+f^{2}\right)^{2}}=-\frac{2 m^{2} \sinh ^{2} \eta-n^{2}}{m^{2} \sinh ^{2} \eta+n^{2}} \frac{\cosh \eta}{\sinh \eta} .
$$

It can be integrated and we find that

$$
\frac{\sigma^{2 / 3} f f^{\prime}}{\left(1+f^{2}\right)^{2}}=\frac{k_{1}}{|m|^{3}} \frac{\sinh \eta}{\left(\frac{n^{2}-m^{2}}{m^{2}}+\cosh ^{2} \eta\right)^{3 / 2}},
$$

where $k_{1}$ is a constant.

Finally for any dielectric function, we obtain the general solution given by the integral

$$
\int \frac{\sigma^{2 / 3} f}{\left(1+f^{2}\right)^{2}} d f=\frac{-k_{1}}{|m|\left(m^{2}-n^{2}\right)} \frac{\cosh \eta}{\left(\frac{n^{2}-m^{2}}{m^{2}}+\cosh ^{2} \eta\right)^{1 / 2}}-\frac{k_{2}}{2},
$$

where $k_{2}$ is the second integration constant. Here, the case $|m|>|n|$ has been assumed.

Before we specify the dielectric function and present exact solution let us consider the total energy of the solution given by (15). In case of our model, the total energy reads

$$
E \equiv \int d^{3} x T_{00}=8^{3 / 4} \int d^{3} x \sigma\left(u, u^{*}\right) \frac{\left(K_{i} \partial^{i} u^{*}\right)^{\frac{3}{4}}}{\left(1+|u|^{2}\right)^{3}} .
$$

Using (12) we get

$$
E_{m, n}=(2 \pi)^{2} 8 \cdot 2^{3 / 4} \int_{0}^{\infty} \frac{d \eta \sinh \eta}{\left(1+f^{2}\right)^{3}}\left(m^{2}+\frac{n^{2}}{\sinh ^{2} \eta}\right)^{\frac{3}{4}} f^{\frac{3}{2}} f^{\prime \frac{3}{2}} \sigma(f) .
$$

Quite surprisingly this expression can be integrated for any dielectric function. In fact, using equation (14) we are able to remove $\sigma$ from (17). Then

$$
E_{m, n}=(2 \pi)^{2} 8 \cdot 2^{3 / 4}\left|k_{1}\right|^{\frac{3}{2}} \int_{0}^{\infty} \frac{d \eta}{\sinh ^{2} \eta}\left(m^{2}+\frac{n^{2}}{\sinh ^{2} \eta}\right)^{-\frac{3}{2}}
$$


Finally, we obtain that

$$
E_{m, n}=(2 \pi)^{2} 8 \cdot 2^{3 / 4} \frac{\left|k_{1}\right|^{\frac{3}{2}}}{|m||n|(|m|+|n|)} .
$$

The total energy is given only in terms of the integer numbers $m, n$ and the first integration constant $k_{1}$. This expression strongly depends on the asymptotic value of the function $f$ i.e. behavior of the unit vector field in origin and at the spatial infinity.

In order to find exact solution one has to choice a particular form of the dielectric function and fix the asymptotic conditions. Let us consider the following family of functions label by the continuous parameter $\delta>-\frac{3}{2}$

$$
\sigma(\vec{n})=\left(1-n^{3}\right)^{\delta} \alpha(\delta)
$$

The new constant is chosen in the form

$$
\alpha(\delta)=\frac{1}{2^{\delta}}\left(\frac{3+2 \delta}{3}\right)^{\frac{3}{2}}
$$

One can notice that a very similar dielectric function appears in the nonAbelian color dielectric model in the limit where dielectric field condenses on some non-trivial value [14].

This dielectric function can be expressed in terms of $f$ as

$$
\sigma=\alpha(\delta)\left(\frac{2}{\left(1+f^{2}\right)}\right)^{\delta}
$$

Then, the integral (15) can be evaluated and we find

$$
\frac{1}{1+f^{2}}=\left[\left(\frac{2 k_{1}}{|m|\left(m^{2}-n^{2}\right)} \frac{\cosh \eta}{\left(\frac{n^{2}-m^{2}}{m^{2}}+\cosh ^{2} \eta\right)^{1 / 2}}+k_{2}\right)\right]^{\frac{3}{2 \delta+3}} .
$$

To calculate unknown constants $k_{1}, k_{2}$ one has to fix the asymptotic conditions. We take

$$
\vec{n} \rightarrow(0,0,1) \text { i.e. } f \rightarrow \infty \text { as } \eta \rightarrow 0
$$

and

$$
\vec{n} \rightarrow(0,0,-1) \text { i.e. } f \rightarrow 0 \text { as } \eta \rightarrow \infty \text {. }
$$

Thus, after some simply algebra one can obtain

$$
k_{1}=\frac{1}{2}\left(m^{2}-n^{2}\right) \frac{|m||n|}{|n|-|m|}
$$


and

$$
k_{2}=\frac{|m|}{|m|-|n|} .
$$

Inserting the constants in (22) we derive that

$$
\frac{1}{1+f^{2}}=\left[\frac{1}{|m|-|n|}\left(|m|-|n| \frac{\cosh \eta}{\left(\frac{n^{2}}{m^{2}}+\sinh ^{2} \eta\right)^{1 / 2}}\right)\right]^{\frac{3}{2 \delta+3}} .
$$

Finally, the function $f$ is found to be

$$
f^{2}=\frac{\left(\left(\frac{|m|}{|n|}-1\right) \sqrt{\frac{n^{2}}{m^{2}}+\sinh ^{2} \eta}\right)^{\frac{3}{2 \delta+3}}-\left(\sqrt{1+\frac{m^{2}}{n^{2}} \sinh ^{2} \eta}-\cosh \eta\right)^{\frac{3}{2 \delta+3}}}{\left(\sqrt{1+\frac{m^{2}}{n^{2}} \sinh ^{2} \eta}-\cosh \eta\right)^{\frac{3}{2 \delta+3}}} .
$$

Knowing the value of the constants we can obtain the total energy corresponding to the solution. It can be checked that

$$
E_{m, n}=(2 \pi)^{2} 4 \cdot 2^{1 / 4} \sqrt{|m||n|(|m|+|n|)} .
$$

Of course, more complicated dielectric function will bring us to more complex solution. We would like to mention only two functions which, in our opinion, lead to quite nice solutions. Namely, for

$$
\sigma_{1}(\vec{n})=\frac{a}{\left(1-n^{3}\right)^{3}} e^{-a \frac{3}{2}\left(\frac{1+n^{3}}{1-n^{3}}\right)}
$$

we get

$$
f^{2}=-\frac{1}{a} \ln \left[\frac{1}{|m|-|n|}\left(|m|-|n| \frac{\cosh \eta}{\sqrt{\frac{n^{2}}{m^{2}}+\sinh ^{2} \eta}}\right)\right],
$$

whereas for

$$
\sigma_{2}(\vec{n})=\frac{8}{\left(1-n^{3}\right)^{3}}\left[\frac{\sinh \left(\frac{1+n^{3}}{1-n^{3}}\right)}{\cosh ^{2}\left(\frac{1+n^{3}}{1-n^{3}}\right)}\right]^{\frac{3}{2}}
$$

one finds

$$
f^{2}=\operatorname{arcosh}\left[\frac{|m|-|n|}{\left(|m|-|n| \frac{\cosh \eta}{\sqrt{\frac{n^{2}}{m^{2}}+\sinh ^{2} \eta}}\right)}\right] .
$$


Both solutions correspond to the same constants $k_{1}, k_{2}$ and possess identical total energy given by (29).

Let us now calculate the Hopf index for previously obtained field configurations. It will be done for the solution (28) but the presented procedure, based on [16], can be easily repeated in case of solutions (31), (33) as well. We introduce two additional functions

$$
g_{1}^{2}=\left(\left(\frac{|m|}{|n|}-1\right) \sqrt{\frac{n^{2}}{m^{2}}+\sinh ^{2} \eta}\right)^{\frac{3}{2 \delta+3}}-\left(\sqrt{1+\frac{m^{2}}{n^{2}} \sinh ^{2} \eta}-\cosh \eta\right)^{\frac{3}{2 \delta+3}}
$$

and

$$
g_{2}^{2}=\left(\sqrt{1+\frac{m^{2}}{n^{2}} \sinh ^{2} \eta}-\cosh \eta\right)^{\frac{3}{2 \delta+3}} .
$$

Then we can define functions $\Phi_{i}, i=1 . .4$

$$
\Phi_{\left(\begin{array}{l}
1 \\
2
\end{array}\right)}=\left(\frac{g_{1}}{\sqrt{g_{1}^{2}+g_{2}^{2}}}\right) \times\left(\begin{array}{c}
\cos m \xi \\
\sin m \xi
\end{array}\right)
$$

and

$$
\Phi_{\left(\begin{array}{l}
3 \\
4
\end{array}\right)}=\left(\frac{g_{2}}{\sqrt{g_{1}^{2}+g_{2}^{2}}}\right) \times\left(\begin{array}{l}
\cos n \phi \\
-\sin n \phi
\end{array}\right),
$$

which are connected with primary unit vector field by the relation $n_{i}=$ $Z^{\dagger} \sigma_{i} Z$, where

$$
Z=\left(\begin{array}{c}
Z_{1} \\
Z_{2}
\end{array}\right), \quad Z^{\dagger}=\left(Z_{1}^{*}, Z_{2}^{*}\right)
$$

and

$$
Z_{1}=\Phi_{1}+i \Phi_{2}, \quad Z_{2}=\Phi_{3}+i \Phi_{4} .
$$

The crucial point is to find Abelian vector field which defines the field tensor $H_{i j}=\partial_{i} A_{j}-\partial_{j} A_{i}$. It can be achieved for the following potential

$$
A_{i}=\frac{i}{2}\left(Z^{\dagger} \partial_{i} Z-\partial_{i} Z^{\dagger} Z\right)
$$

The Hopf index is defined in the standard way as

$$
Q_{H}=\frac{1}{4 \pi^{2}} \int d^{3} x \vec{A} \cdot \vec{B}
$$

where 'magnetic' field $\vec{B}=\nabla \times \vec{A}$. After integration we find that

$$
Q_{H}=\frac{n m}{2}\left[\left(\Phi_{1}^{2}+\Phi_{2}^{2}\right)^{2}-\left(\Phi_{3}^{2}+\Phi_{4}^{2}\right)^{2}\right]_{0}^{\infty}=-m n .
$$


For non-zero $m, n$ numbers the presented solutions possess the non-trivial Hopf index. We would like notice that the famous Vakulenko-Kapitansky non-equality [20], also in the case where $O(3)$ symmetry is explicitly broken, is fulfilled. In fact, we find that

$$
E_{m, n} \geq(2 \pi)^{2} 4 \cdot 2^{\frac{3}{4}}\left|Q_{H}\right|^{\frac{3}{4}} .
$$

One can see that we reproduce the result recently found in the symmetric case [16].

\section{Conclusions}

In the present paper $O(3)$ symmetry breaking models built of the unit vector field leaving in the four dimensional Minkowski space-time have been investigated. In general, the Lagrangian consists of two terms. The first one, global $O(3)$ symmetric, depends on the $H_{\mu \nu}^{2}$ is multiplied by a dielectric function $\sigma(\vec{n})$. This function ensures the explicit symmetry breaking on the Lagrangian level.

Let us summarized the achieved results.

First of all, it has been proved that such models are integrable in this sense that infinite number of the conserved currents can be constructed. As we have mentioned it before, integrability of models with broken global symmetry is rather exceptional effect if we compare it with the standard theories with solitons. Usually, integrability as well as appearance of solitons requires the maximal possible symmetry.

Secondly, the special case, where the $O(3)$ symmetric term is chosen in the form to circumvent the famous Derick scaling argument for non-existence of stable solitons, has been analyzed in details. We have found a family toroidal solutions (in general given by the integral), label by two integer numbers $m, n$. The total energy has been also found. It is given by these numbers and the first integration constant i.e. by the asymptotic value of the field. For some particular dielectric functions the exact solutions have been obtained. For the most interesting case where the dielectric function has a form motivated from non-Abelian color dielectric model [14, the generalized Aratyn-Ferreira-Zimerman solutions have been presented. We have also proved that these field configurations possess non-trivial topology and can be classified by means of the Hopf index. Quite intriguing, the energycharge inequality takes identical form as in the symmetric case.

The main result of our work is that toroidal Hopf solitons can appear in the model with broken global $O(3)$ symmetry. It should be stressed that the $O(3)$ global symmetry is not essential for existence of hopfions. 
It is also worth to notice that analyzed here pattern of the symmetry breaking (i.e. via the dielectric function) is a very special one. The breaking of the symmetry is reflected merely in the shape of the soliton. In spite of the fact that the form of the soliton solutions strongly depends on the dielectric function and can be really complicated, they all lead to the same total energy and Hopf index as in the symmetric theory. Due to that our model can be understood as the model where the $O(3)$ symmetry breaking occurs in the minimal way.

The existence of topological toroidal solutions in the model with the explicit broken symmetry is very encouraging in the context of the effective model for the low energy gluodynamics. As we have mentioned, there are some numerical [13] as well as theoretical work [11, 12], 14] which suggest that such effective model should break global $O(3)$ symmetry. In fact a model model with symmetry breaking dielectric function has been recently proposed [14. Because of the fact that symmetry breaking in this model occurs in the same manner as considered here one can suppose that also for the model [14] the influences from the breaking terms may be insignificant. It would be very interesting to investigate it more precisely. We plan to address this problem in our proceeding paper.

This work is partially supported by Foundation for Polish Science and ESF "COSLAB" programme.

\section{References}

[1] L. Faddeev and A. J. Niemi, Nature 387, 58 (1997); L. Faddeev and A. J. Niemi, Phys. Rev. Lett. 82, 1624 (1999).

[2] E. Langmann, A. Niemi, Phys.Lett. B 463, 252 (1999).

[3] Y. M. Cho, Phys.Rev.D 21, 1080 (1980); Phys.Rev. D 23, 2415 (1981).

[4] D. A. Nicole, J. Phys. G 4, 1363 (1978).

[5] R. A. Battye and P. M. Sutcliffe, Phys. Rev. Lett. 81, 4798 (1998); R. A. Battye and P. M. Sutcliffe, Proc.Roy.Soc.Lond. A 455, 4305 (1999).

[6] J. Hietarinta and P. Salo, Phys. Lett. B 451, 60 (1999); J. Hietarinta and P. Salo, Phys. Rev. D 62, 81701 (2000).

[7] R. S. Ward, Phys.Lett.B 473, 291 (2000); R. S. Ward, hep-th/9811176.

[8] P. van Ball, A. Wipf, Phys.Lett. B 515, 181 (2001). 
[9] L. Faddeev, A. J. Niemi, U. Wiedner, hep-ph/0308240 A. J. Niemi, hep-th/0312133.

[10] M. Iwasaki, S. Nawa, T. Sanada, F. Takagi, Phys. Rev. D 68, 074007 (2003); J. P. Ralston, hep-ph/0301089.

[11] L. Faddeev, A. J. Niemi, Phys. Lett. B 525, 195 (2002).

[12] J. Sánchez-Guillén, Phys.Lett. B 548, 252 (2002), Erratum-ibid. B 550, 220 (2002); J. Sánchez-Guillén, hep-th/0211277.

[13] L. Dittmann, T. Heinzl, A. Wipf, Nucl. Phys. B (Proc. Suppl.) 106, 649 (2002); Nucl. Phys. B (Proc. Suppl.) 108, 63 (2002).

[14] A. Wereszczyński, M. Ślusarczyk, hep-ph/0405148.

[15] H. Arodź, H. J. Pirner, Acta Phys. Pol. B 30, 3895 (1999).

[16] H. Aratyn, L. A. Ferreira and A. H. Zimerman, Phys. Lett. B 456, 162 (1999); H. Aratyn, L. A. Ferreira and A. H. Zimerman, Phys. Rev. Lett. 83, 1723 (1999).

[17] C. Adam and J. Sánchez-Guillén, J.Math.Phys. 44, 5243 (2003).

[18] O. Alvarez, L. A. Ferreira, J. Sánchez-Guillén, Nucl. Phys. B 529, 689 (1998).

[19] O. Babelon and L. A. Ferreira, JHEP 0211, 020 (2002).

[20] A. F. Vakulenko, L. V. Kapitansky, Sov. Phys. Dokl. 24, 432 (1979). 